\title{
Coronal electron acceleration and relativistic proton production during the 14 July 2000 flare and CME
}

\author{
K.-L. Klein ${ }^{1}$, G. Trottet ${ }^{1}$, P. Lantos ${ }^{1}$, and J.-P. Delaboudinière ${ }^{2}$ \\ 1 DASOP, CNRS-UMR 8645, Observatoire de Paris, Section de Meudon, 92195 Meudon, France \\ 2 Institut d'Astrophysique Spatiale, Université Paris-Sud-CNRS, Bât. 121, 91405 Orsay, France
}

Received 6 March 2001 / Accepted 27 April 2001

\begin{abstract}
The large solar flare of 14 July 200010 UT occurred in an active region near the central meridian. It was accompanied by the eruption of a filament and a rapid halo-type coronal mass ejection (CME). Large particle fluxes were detected up to relativistic energies at 1 AU. In this paper accelerated particles and plasma structures in the corona are traced using radio, X-ray, EUV and visible light observations, together with neutron monitor measurements of relativistic protons at $1 \mathrm{AU}$. Both the bulk of the radio emission at decimetric and longer waves and the escape of suprathermal electrons and relativistic protons from the Sun were delayed by 10-20 min with respect to the hard X-ray emission. Despite the delay and the association with a flare near the central meridian the neutron monitor time profile was impulsive. We show that the escape of the relativistic protons occurred in time coincident both with a coronal shock wave, which may be the bow shock of the CME, and with radio sources which trace electron acceleration and magnetic field reconfiguration in the western hemisphere. Three observations support the idea that the relativistic protons were accelerated during this reconfiguration, at heights between 0.1 and $1 R_{\odot}$ above the photosphere, and not in the flaring active region or at the bow shock of the CME: (i) the rise of the neutron monitor count rates is simultaneous with the brightening of a new continuum radio source; (ii) the duration of the continuum emission is similar to the rise time of the neutron monitor count rates; (iii) the radio source is close to the Earth-connected interplanetary magnetic field line.
\end{abstract}

Key words. Sun: activity; flares; particle emission; radio radiation

\section{Introduction}

A large solar event occurred near 10 UT on 14 July 2000, comprising a flare as revealed by bright emission throughout the electromagnetic spectrum, the eruption of a filament, a fast halo-type coronal mass ejection (CME), and a large particle flux enhancement in interplanetary space. Neutron monitors showed a rapid rise of relativistic proton fluxes at $1 \mathrm{AU}$ to the strongest maximum observed since October 1989. Favourable observing conditions at EUV, X-ray and radio wavelengths enable us to study with some detail the evolution of coronal structures and the associated particle acceleration, and to discuss the question of how these processes are related to the acceleration of the relativistic protons eventually observed at $1 \mathrm{AU}$. We thereby pursue the study of individual solar relativistic proton events (called ground level events, GLEs) which suggested that relativistic protons are injected into interplanetary space well after the production of interacting

Send offprint requests to: K.-L. Klein, e-mail: Ludwig.Klein@obspm.fr energetic particles revealed by their gamma-ray and hard $\mathrm{X}$-ray emission, but in time coincidence with acceleration in the middle corona $\left(\sim 0.1-1 R_{\odot}\right.$ above the photosphere, say) that shows up in decimetric and metric radio emission (Klein et al. 1999; Klein \& Trottet 2001). While the delay of relativistic proton injection with respect to hard X-ray, gamma-ray and microwave signatures of mildly relativistic electrons and suprathermal protons in the low solar atmosphere was known earlier (e.g., Carmichael 1962; Cliver et al. 1982; Kahler 1994; Debrunner et al. 1997), the connection with signatures of coronal particle acceleration at greater height, where collisional radiative signatures are inefficient due to the low ambient density, and gyrosynchrotron emission is faint because of the weak magnetic field, has only rarely been investigated (Palmer \& Smerd 1972; Kocharov et al. 1994; Akimov et al. 1996; Klein et al. 1999; Miroshnichenko et al. 2000).

This paper is organised as follows: Sect. 2 starts with an overview of whole-Sun radiation signatures of energetic electrons and the onset of the relativistic proton injection into interplanetary space (2.1). Imaging data at EUV (2.2) 
and long decimetric and short metric waves (2.3) are then presented and put into the context of the CME observations (2.4). These results are discussed in Sect. 3 with respect to the role of coronal acceleration in producing the relativistic protons during this event, as opposed to acceleration at the bow shock of the rapid CME, which is the most frequently invoked mechanism for the acceleration of suprathermal (e.g., Reames 1999, and references therein) and relativistic nucleons (Cliver et al. 1982; Lockwood et al. 1990; Kahler 1994).

\section{Observations}

Shortly after 10 UT on 14 July 2000 a filament eruption and a class X5 (3B) flare occurred in active region AR 9077 near the central meridian. The event was associated with a fast CME (estimated speed $1775 \mathrm{~km} \mathrm{~s}^{-1}$, Fox 2000) and was followed by a rich variety of particle signatures in interplanetary space and a geomagnetic storm.

\subsection{Whole Sun $X$-ray and radio emission and relativistic proton event}

$\mathrm{X}$-ray and radio time histories of the whole Sun emission are plotted in Fig. 1, together with the rise of the count rates at some neutron monitors.

The soft X-ray event (second panel from bottom) was of moderately long duration (about $1.1 \mathrm{hr}$ at the $10 \%$ of maximum level). The rise to maximum (10:24:09 UT) within about ten minutes was followed by a decay which flattened after 10:25 and displayed a bump. The derivative of the soft X-ray flux (dashed line in the second panel from bottom) suggests that the main phase of energy conversion in the low corona lasted from about 10:03 to 10:24 UT, with a maximum near 10:18-10:19 UT.

Hard X-rays (Fig. 1, bottom) were observed at the beginning of the event by the HXRS experiment aboard the Multispectral Thermal Imager (MTI) satellite, at the end by HXT/Yohkoh. Comparison with the derivative of the soft $\mathrm{X}$-ray time profile suggests that the maximum was missed by both hard X-ray instruments. HXT saw bursts superposed on a prolonged decay between 10:20 and 10:40 UT. The bump in the post-maximum soft $\mathrm{X}$-ray flux curve coincided with a new episode of energy release traced by the hard X-ray burst between 10:25 and 10:30 UT.

The arrival of relativistic protons at Earth is traced by the neutron monitor count rates in the top panel of Fig. 1. The abscissa gives the arrival time at the detector minus $150 \mathrm{~s}$. The $150 \mathrm{~s}$ difference removes the prolonged travel time, with respect to photons, of relativistic charged particles along an interplanetary magnetic field line with assumed length 1.3 AU. Their injection into interplanetary space lagged the onset of the hard X-ray burst by about $20 \mathrm{~min}$, and its presumed maximum by more than 10 min. Hard X-ray emission was smoothly fading when the protons were released. The GLE increase was detected in the same time interval (10:34-10:35 UT for a
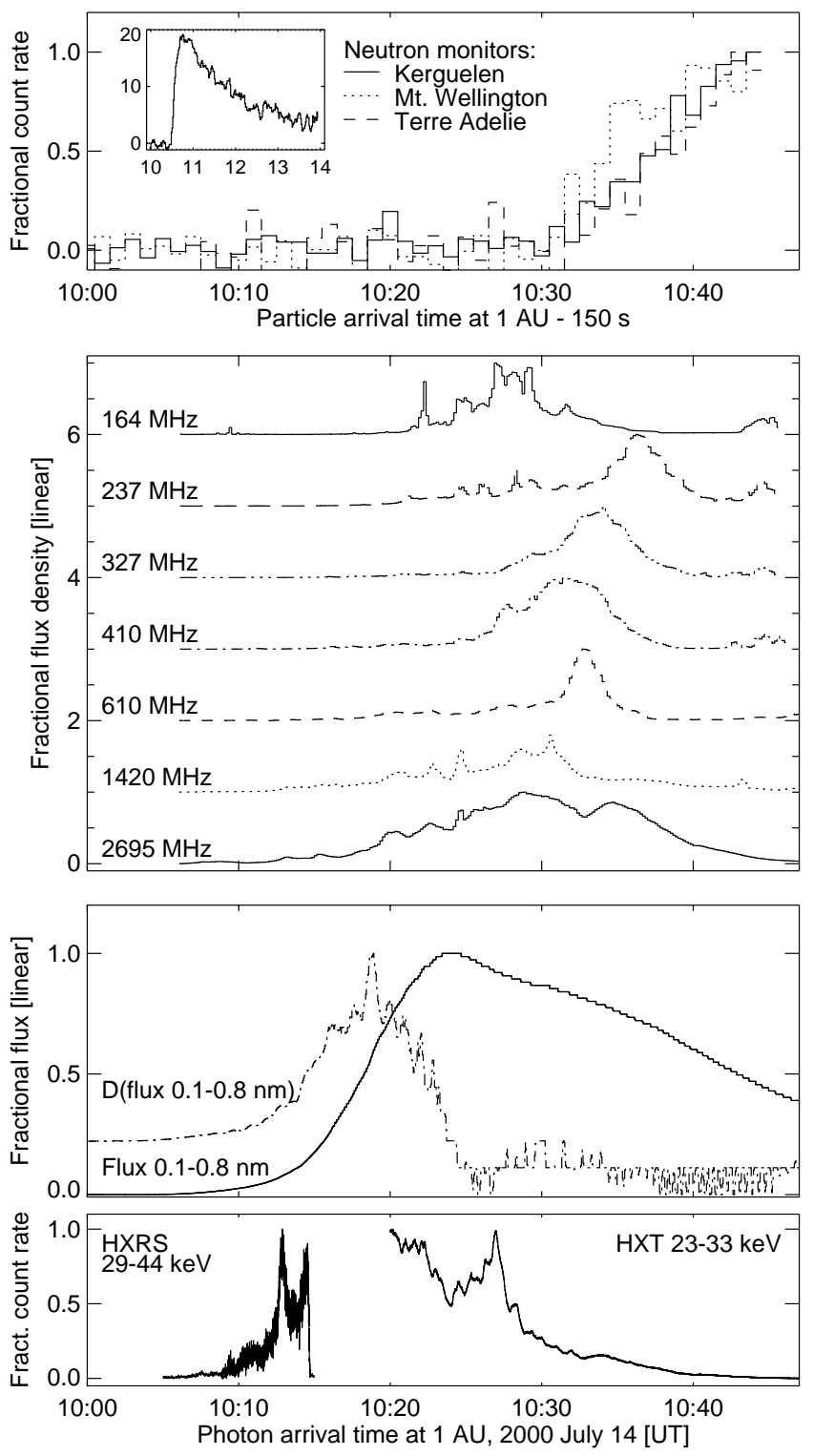

Fig. 1. Time history of X-ray and radio emission compared with neutron monitor data. From bottom to top: hard Xrays (HXRS, courtesy F. Farnik; HXT/Yohkoh, courtesy J. I. Khan), soft-rays (1-8 $\AA$, GOES: solid line) and their time derivative (dashed), radio emission at decimetric and metric wavelengths (Trieste, courtesy P. Zlobec; NRH) and neutron monitor signal. The time axis of the latter gives the detection time minus $150 \mathrm{~s}$ to compensate for the longer distance relativistic charged particles have to travel along the interplanetary magnetic field. The percentage increase at Mt. Wellington is plotted in the inset.

$3 \sigma$ excess above background) by the four stations we examined (Terre Adélie, Kerguelen, Mount Wellington and Hobart). Signals at Hobart and Mount Wellington rose from background to maximum within 10-15 min, which is faster than at the other two monitors. This is evidence for anisotropy of the protons arriving at $1 \mathrm{AU}$, while the decay was isotropic (similar decay for the four stations). The neutron monitors are accessible to protons with energies 


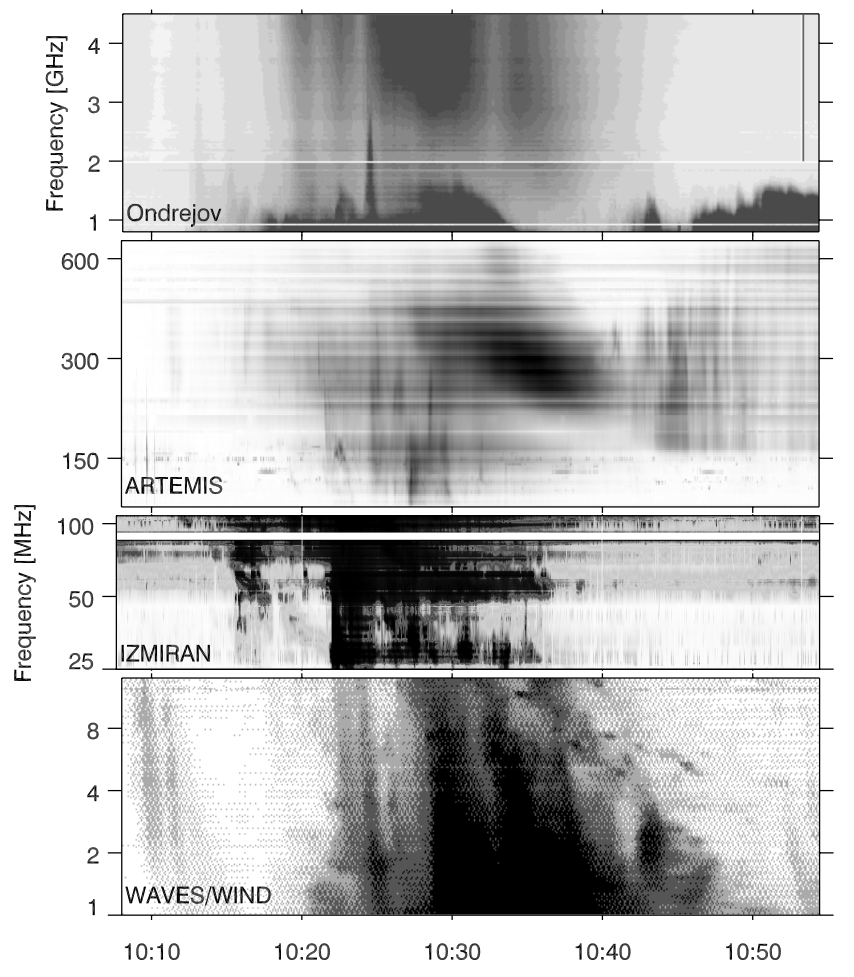

Fig. 2. Radio spectra from microwaves to hectometric waves during the 14 July 2000 event. From top to bottom: Ondřejov Observatory (Karlický et al. 2001), Artemis-IV, Thermopylae, Greece (courtesy C. Alissandrakis; Caroubalos et al. 2001), IZMIRAN, WAVES/Wind (courtesy M. Kaiser). Dark shading means bright emission.

exceeding $450 \mathrm{MeV}$ (Terre Adélie), $510 \mathrm{MeV}$ (Kerguelen), and $1.1 \mathrm{GeV}$ (Mt. Wellington, Hobart), respectively.

Decimetric and metric radio emission (Fig. 1, third panel from bottom) started near the onset of the hard X-ray event, but the bulk was delayed by more than ten minutes. The dominant feature between 410 and $237 \mathrm{MHz}$ drifted slowly towards lower frequencies ( 10:30-10:40 UT). Dynamic spectra of the main radio emission from a few $\mathrm{GHz}$ to $1 \mathrm{MHz}$ are plotted in Fig. 2 . Weak emission was detectable from $\sim 10: 10$, i.e. during the rise of the hard X-rays. A few faint type III bursts were observed at that time at metric (cf. $150 \mathrm{MHz}$ ) and hectometric wavelengths. The bulk of the radio emission between 10:20 and 10:40 UT had different spectral components: simultaneously starting and ending broadband emission at frequencies above $2 \mathrm{GHz}$, burst-like emission from $2 \mathrm{GHz}$ down to at least $1 \mathrm{MHz}$, and a bright, structureless continuum ( 450-200 MHz; 10:30-10:40 UT) with a slow drift towards lower frequencies. This continuum was the prominent feature in the time profiles between 410 and $237 \mathrm{MHz}$ in Fig. 1. Despite its slow frequency drift, this spectral feature was not a type II burst: it had a much larger instantaneous bandwidth than type II bursts, and lacked intrinsic spectral fine structure such as fundamental/harmonic bands, splitbands, or herringbone bursts.

The continuum was accompanied by a group of intense type III bursts at dekametric and hectometric wavelengths, with a sudden onset near 10:22 UT, and a particularly bright phase between $\sim 10: 30$ and 10:40 UT (bottom panel, Fig. 2). During this bright phase the starting frequency of the type III bursts drifted gradually to lower frequencies. Narrowband drifting emission was visible near the starting frequency (e.g., 10:35-10:45 UT between $14 \mathrm{MHz}$ and $5 \mathrm{MHz}$ ), but the detailed relationship was obscured by the obervational gap between $25 \mathrm{MHz}$ and $14 \mathrm{MHz}$. The narrowband emission was part of a hectometric to kilometric type II burst. This reveals a shock wave. Given the low frequency, the shock wave was most probably well above the sources of decimetric and metric radio emission.

After 10:40 UT, a new broadband emission with burst-like structure was observed between $1.5 \mathrm{GHz}$ and $\sim 150 \mathrm{MHz}$. The decimetric emission during the 14 July and preceding events is discussed in detail by Karlický et al. (2001).

In summary, the time histories at radio, hard and soft $\mathrm{X}$-ray wavelengths suggest several distinct episodes of energy release during the event. The low coronal energy release as traced by the hard X-rays and the main rise of the soft X-rays was accompanied only by relatively faint radio emission, including weak hectometric type III bursts near 10:10 UT. Energy release within a much larger volume was revealed thereafter by intense decimetric to hectometric radio emission during the decay of the X-ray signatures. The brightest radio emission at all frequencies occurred together with the decimetric/metric continuum, with the bump during the decay of the soft X-ray burst, and with the tail of hard X-ray emission. Electrons accelerated by a coronal shock wave at higher altitude were simultaneously traced by hectometric type II emission.

Relativistic protons were injected into interplanetary space during this phase of intense decimetric to hectometric radio emission. This time coincidence does not critically depend on the assumed path length of protons along the interplanetary magnetic field. If, instead of $1.3 \mathrm{AU}$ as assumed in Fig. 1, it were 1.1 AU or 1.5 AU, the neutron monitor time profiles would have to be shifted by $\pm 100 \mathrm{~s}$.

\subsection{Imaging observations at EUV wavelengths}

The main flare signatures at optical and EUV wavelengths were seen in AR 9077 north of the equator between heliographic longitudes $-5^{\circ}$ and $+15^{\circ}$. The magnetic field configuration in the active region is discussed by Kosovichev \& Zharkova (2001) and Yan et al. (2001). Figure 3 displays the sequence of whole-Sun images observed by EIT on SoHO (Delaboudinière et al. 1995). Difference images are used to make faint features more readily visible. The detailed evolution within the flaring active region was observed by TRACE (cf. TRACE movies, e.g. Title 2000).

The first signatures of the event were related to the eruption of a filament (arrow in Fig. 3c) in the leading part of AR 9077 between 10:00:10 and 10:12:52. It was located east of $\mathrm{W} 10^{\circ}$ before its eruption. Subsequently bright 

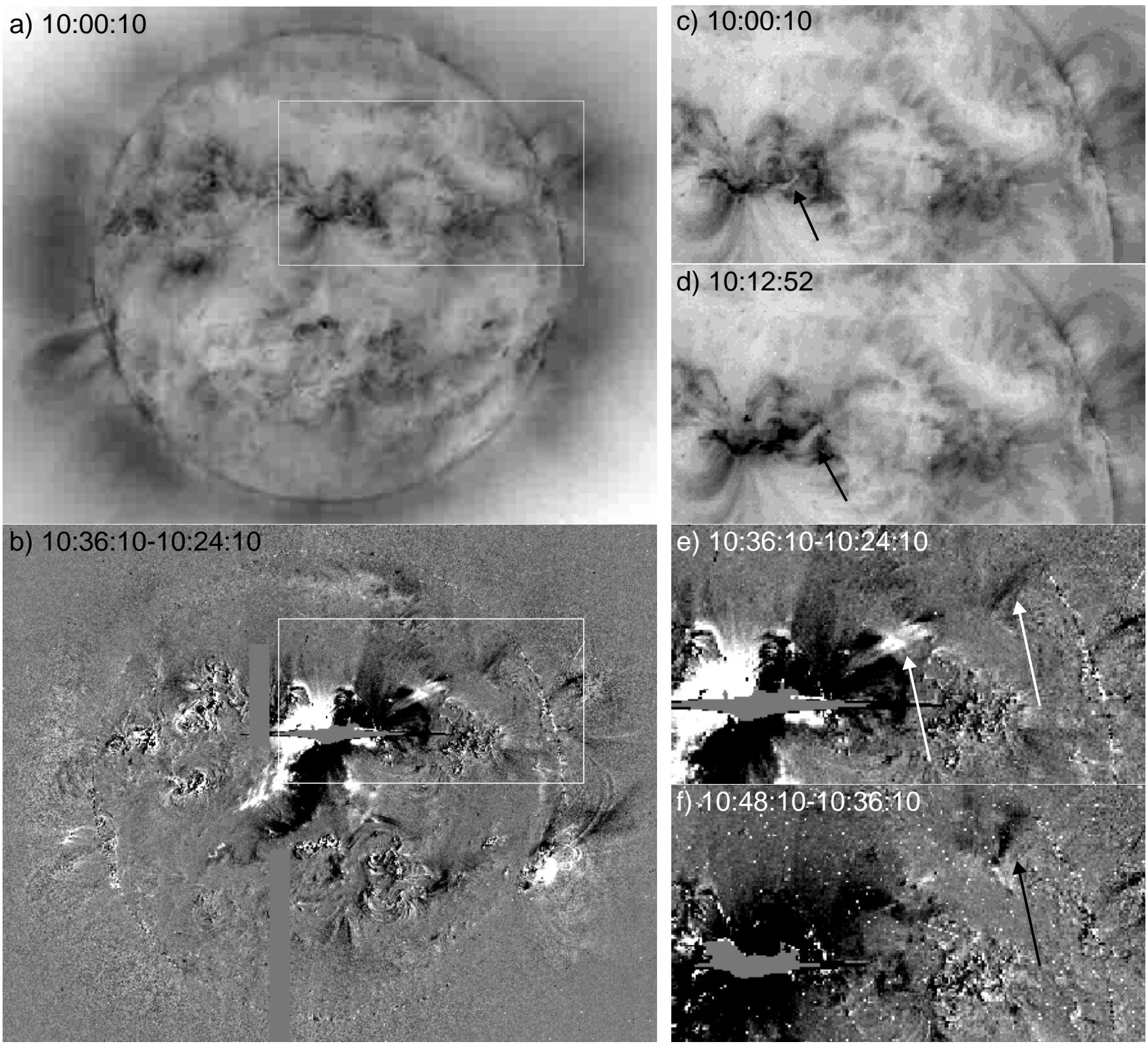

Fig. 3. The flare and CME of 14 July 2000 as seen by EIT/SoHO at $195 \AA$ (solar north up, west to the right). Left column: whole Sun negative image before the event a) and difference between images taken at 10:36:10 and 10:24:10 b). The white rectangle delimits the field plotted in the right column. Uniformly grey areas designate regions where data are missing (two vertical rectangles east of central meridian in b) or where the detector was saturated (ragged area in the flaring active region). The arrows in $\mathbf{c}$ )-e) point to the erupting filament. It is seen in e) at its 10:24:10 position (white shading, positive difference in regions where the filament appeared in absorption at that time) and at its 10:36:10 position (black shading). In f) dimmings persist in the region where the filament had been at 10:36:10 (black arrow).

emission developed around the former location of the filament. It saturated the EIT detector and created a broad diffuse brightening around AR 9077 due to straylight. In the TRACE EUV movie the emission was restricted to the western half of the active region until $\sim 10: 25 \mathrm{UT}$. Afterwards activity continued in the western part of the active region, and spread to its eastern (trailing) part, where flare ribbons and a loop arcade developed. The eastward progression of the brightening took a few minutes. It shows up in the difference image in Fig. 3b as the white shading (i.e. excess emission) around the trailing part and the black shading around the leading part of AR 9077. The sources of the hard X-ray emission observed by YohkohHXT were located at the footpoints of the EUV loops (cf. Yohkoh-homepage; L. Fletcher, pers. comm.).

The motion of the ejected filament through the corona is traced by the EIT $195 \AA$ image at 10:12:52 and the subsequent difference images in Figs. $3 \mathrm{~d}-\mathrm{f}$ : in the difference of the images at 10:36:10 and 10:24:10 (Fig. 3e) the filament appears bright at the position occupied at 10:24:10 

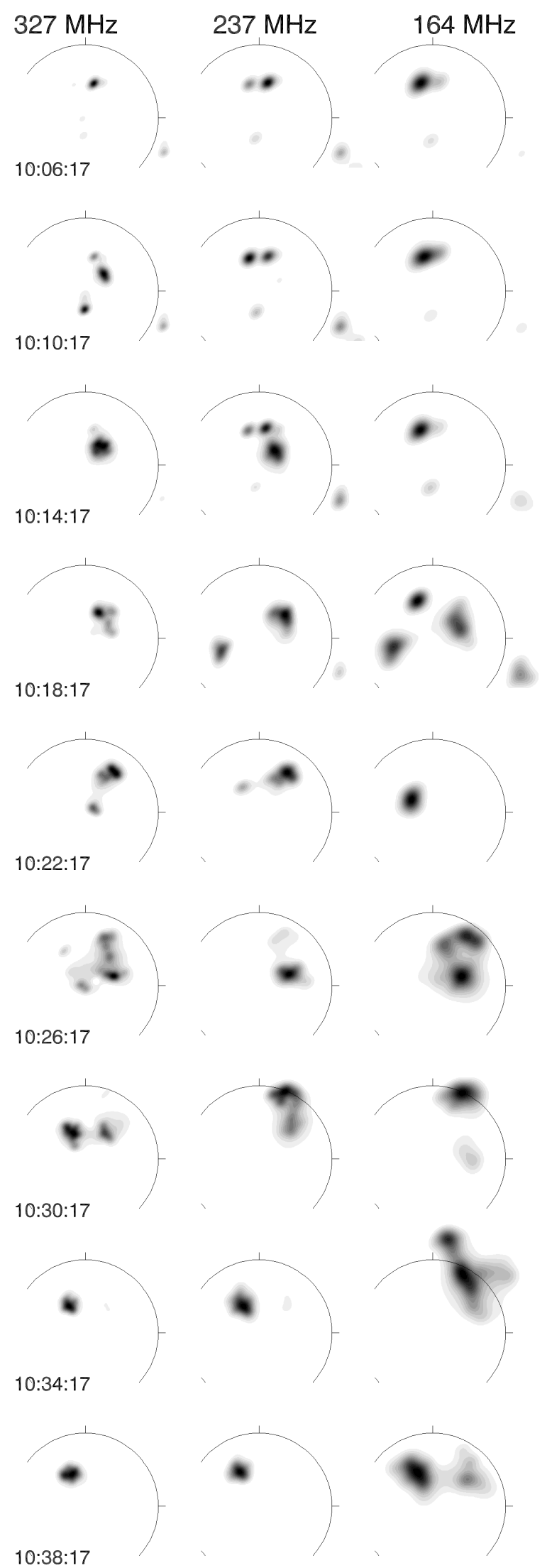

Fig. 4. NRH snapshot maps at $4 \mathrm{~min}$. intervals during the 14 July 2000 event. Dark shading means high brightness. Only levels above the average of each map are plotted. Each map is normalised to its maximum. North is up, west to the right. and dark at its 10:36:10 location. Figure 3f still shows a dimming in the region where the filament was at 10:36, with a greater spatial extent. This dimming is not the filament itself, but gives evidence that coronal structures were modified by its passage, implying interaction between the magnetic field that confines the filament and pre-existing magnetic field structures in the western hemisphere. The ejected filament crossed the north-western solar quadrant at projected speeds of $250 \mathrm{~km} \mathrm{~s}^{-1}$ and $360 \mathrm{~km} \mathrm{~s}^{-1}$ between 10:12:52 and 10:24:10 and 10:24:10 and 10:36:10, respectively.

Besides the filament, a large scale perturbation was seen all over the north-western quadrant. Faint coronal structures around the northern pole were perturbed. This created the changes seen near the pole in Fig. 3b. Temporary dimmings appeared in various regions of the north-western solar quadrant. Remarkably, the quiescent filament and prominence near the north-western limb (Fig. 3a) remained unaffected.

\subsection{Imaging observations at decimetric and metric radio waves}

The time evolution of the radio source configuration at long decimetric and short metric wavelengths is illustrated in the series of maps taken by the Nançay Radioheliograph (NRH; Kerdraon \& Delouis 1997) in Fig. 4. The first row shows the pre-flare configuration, where the dominant emission came from a pair of noise storms above AR 9077 that was observed since the beginning of the NRH observations at 08:27 UT. Fainter noise storm sources were associated with active regions in the southern hemisphere near central meridian and at the south-western limb.

As the EUV images, the radio maps show activity which was initially connected to the leading part of AR 9077 (Fig. 4: cf. 10:14:17 at 327 and $237 \mathrm{MHz}$, 10:18:17 at $164 \mathrm{MHz}$ ) and later shifted to the trailing part. Complex sources were observed in the north-western solar quadrant, as well as brightenings in the eastern hemisphere and in the noise storm above the western limb (cf. 10:18:17 at 237 and $164 \mathrm{MHz}$ ). Before the onset of the drifting continuum the radio emission consisted of groups of type III-like bursts on top of a diffuse background (Fig. 2). The drifting continuum emission came first from a source near the leading part of the active region (e.g., 10:30:17 at $327 \mathrm{MHz}, 10: 38: 17$ at $164 \mathrm{MHz}$ in Fig. 4), and then gradually shifted to a distinct source in the trailing part. At $164 \mathrm{MHz}$ the western continuum source was weak, but nonetheless visible in the maps (e.g., 10:38:17 in Fig. 4). The eastern source became gradually dominant at all frequencies and persisted during several hours with continuing structural changes. During the continuum at high frequencies, moving type IV sources were observed at 237 and $164 \mathrm{MHz}$. The prominent sources at 237 and $164 \mathrm{MHz}$ in the 10:30:17 map of Fig. 4 show the moving type IV burst near its onset. 

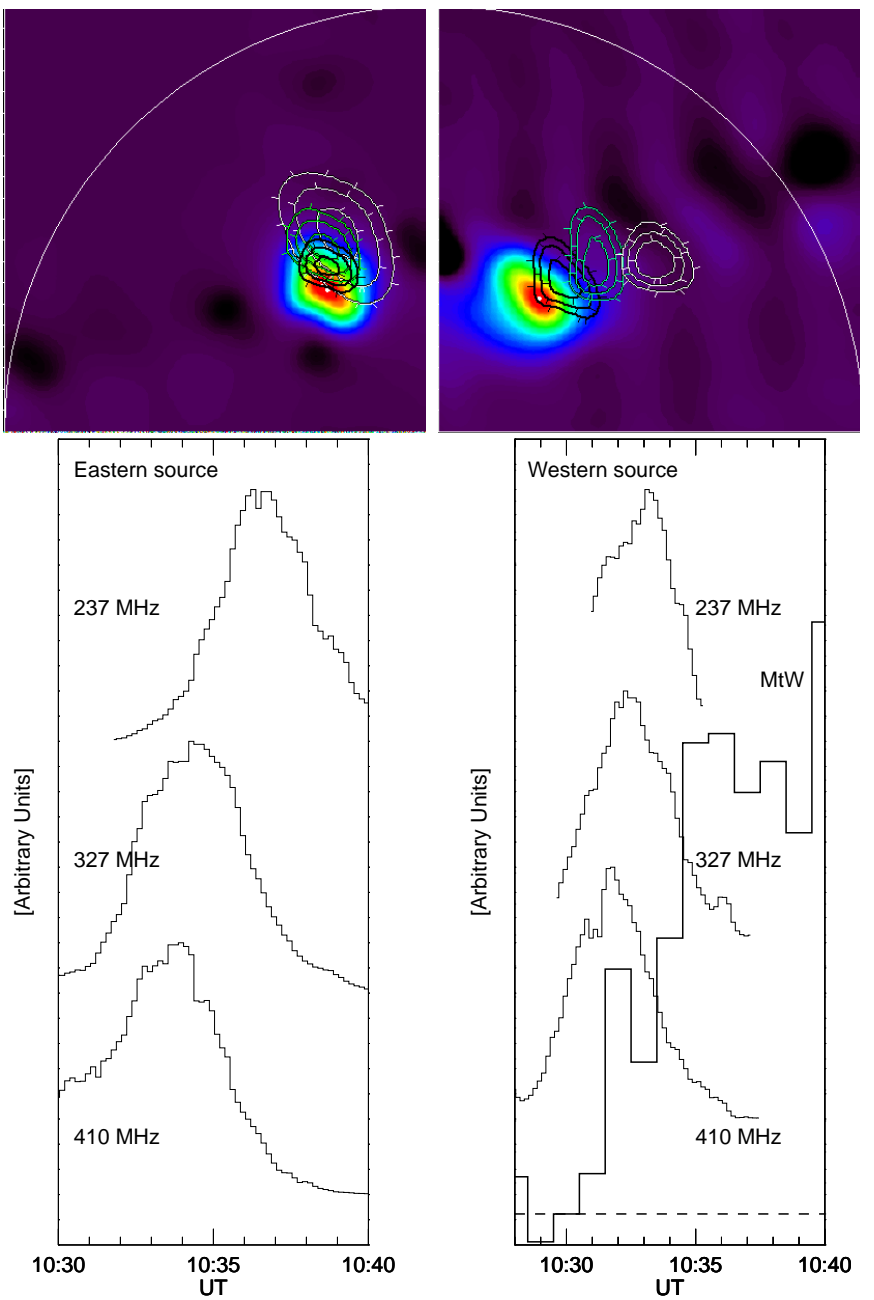

Fig. 5. Multi-frequency maps (top) and time evolution of the western (bottom right) and eastern source (left) of the drifting continuum. The maps show the sources near their maximum brightness at $410 \mathrm{MHz}$ (greyscale), 327, 237 and $164 \mathrm{MHz}$ (contours of equal brightness temperature, from black to white in the order of decreasing frequency). Dark patches are negative sidelobes due to the strong eastern continuum source. The initial rise of the Mount Wellington neutron monitor is overplotted on the brightness temperature profile of the western continuum source, with the usual correction for the presumed travel time diffference of $150 \mathrm{~s}$. The horizontal dashed line is the background of the neutron monitor signal.

In the following we focus on the drifting continuum and the moving type IV burst, which occurred simultaneously with the injection of suprathermal electrons and relativistic protons into interplanetary space.

\subsubsection{The decimetric-metric continuum}

The two radio sources in the leading and the trailing part of the active region brightened sequentially, and both were gradually delayed at low frequencies. The time histories of the peak brightness temperatures are plotted in Fig. 5 (bottom). For both sources the time profiles were similar at the three frequencies, and delayed as a whole at the lower frequencies. The cross correlation coefficient of the time histories is about 0.9 between the different frequencies for each source and between the two sources at each frequency. The frequency drift rates for the western (eastern) source are $-2.8 \mathrm{MHz} \mathrm{s}^{-1}\left(-1.7 \mathrm{MHz} \mathrm{s}^{-1}\right)$ and $-2 \mathrm{MHz} \mathrm{s}^{-1}\left(-0.65 \mathrm{MHz} \mathrm{s}^{-1}\right)$ between 410 and $327 \mathrm{MHz}$ and between 327 and $237 \mathrm{MHz}$, respectively.

Figure 5 (top) shows multifrequency brightness temperature maps of the two continuum sources. The clear frequency dispersion of the western source indicates a coronal magnetic structure which bends westward and towards lower latitudes with decreasing frequencies (i.e. with increasing altitudes). This source was not significantly polarised. Peak brightness temperatures were $3 \times 10^{9} \mathrm{~K}$, $2 \times 10^{10} \mathrm{~K}$ and $2 \times 10^{10} \mathrm{~K}$ at 237,327 and $410 \mathrm{MHz}$ respectively. The high brightness temperature and low degree of polarization of this source are indicative of harmonic plasma emission. Emission from the western source lasted from $\sim 10: 28.5$ (start at $410 \mathrm{MHz}$ ) to $\sim 10: 39$ (end at $164 \mathrm{MHz}$ ).

The eastern source had less pronounced frequency dispersion. The configuration of Fig. 5 suggests that the radio emission arose from a northward or north-westward extending structure anchored in the trailing part of AR 9077. The radiation was left-handed circularly polarised, with a maximum degree of $40 \%$ at 327 and $237 \mathrm{MHz}$, and of $90 \%$ at $410 \mathrm{MHz}$. Its maximum brightness temperature was $6 \times 10^{10} \mathrm{~K}, 10^{11} \mathrm{~K}$ and $3 \times 10^{10} \mathrm{~K}$ at 237,327 and $410 \mathrm{MHz}$, respectively. The brightness temperature and polarisation suggest mostly fundamental plasma emission at $410 \mathrm{MHz}$ and a mixture of fundamental and harmonic plasma emission at the other frequencies.

\subsubsection{Moving type IV emission}

The moving type IV emission was most prominent at $164 \mathrm{MHz}$. Maps during the time interval 10:28-10:38 UT are plotted in Fig. 6. Two sources are seen in the first map. The equatorward source disappeared near 10:31. The northern source moved continuously northward, leaving behind a diffuse faint emission (e.g., 10:32:17). Two sources can be distinguished within the diffuse pattern at 10:33:17. The north-western one was a distinct moving type IV burst with westward direction. The southern source was stationary. It brightened and gradually extended southward, while the two moving sources receded towards north-east and west. Both moving sources were about $50 \%$ right-hand circularly polarised, while the stationary sources were not polarised. The moving sources could be tracked until 10:37. The remaining source at 10:38:17 is the drifting continuum (cf. Fig. 5, top right).

The northward moving sources were similar at 237 and $164 \mathrm{MHz}$. The systematic motion started simultaneously near 10:30 UT, and the projected speeds measured by a linear fit to the trajectories of the centroids of the polarised sources at the two frequencies were, respectively, $1010 \mathrm{~km} \mathrm{~s}^{-1}$ and $1150 \mathrm{~km} \mathrm{~s}^{-1}$. The source probably 


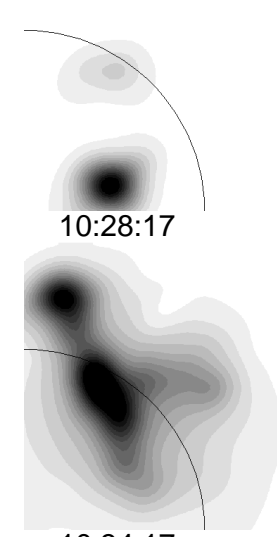

10:34:17

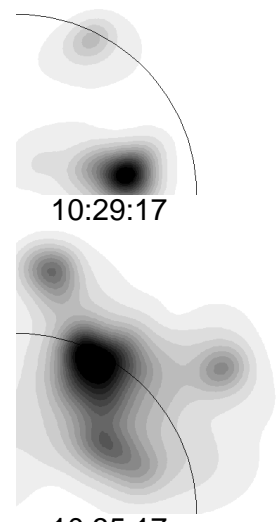

10:35:17

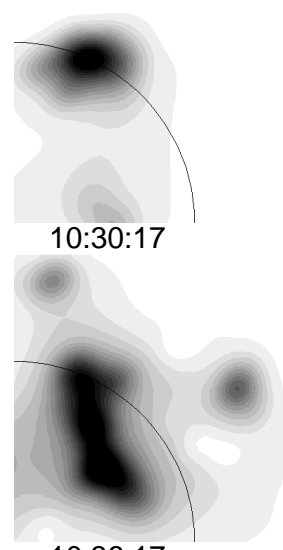

10:36:17
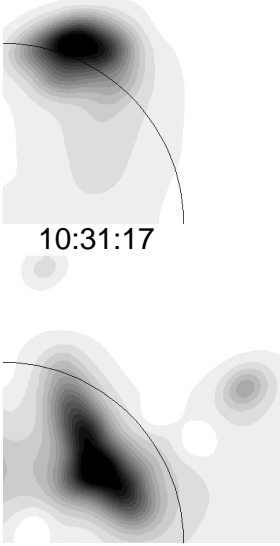

10:37:17

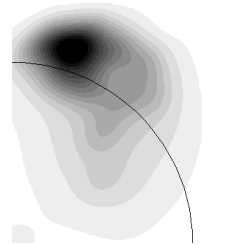

10:32:17

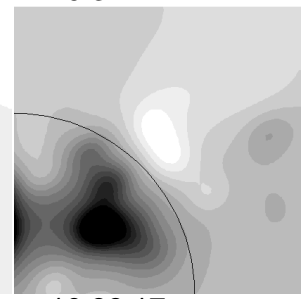

10:38:17

Fig. 6. Snapshot maps (10 s integration) of the north-western solar quadrant during the moving type IV burst at $164 \mathrm{MHz}$. Dark shading means high brightness temperature. Each map is normalised to its maximum brightness temperature. Only levels above the average of the map are plotted. The white patch near the centre of the 10:38:17 map is due to a negative sidelobe of the strong source in the eastern hemisphere (cf. Fig. 4, last row).

contained unresolved components along the east-west direction (Fig. 6, e.g. 10:31:17), which introduce some uncertainty into these values, but the north-south co-ordinates of the sources at 164 and $237 \mathrm{MHz}$ are indistinguishable during the whole period when the moving source was well identified at $237 \mathrm{MHz}$, i.e. between 10:30 and 10:35 UT. The westward moving type IV source was only marginally visible at $237 \mathrm{MHz}$. Its projected speed at $164 \mathrm{MHz}$ was in the range (800-1300) $\mathrm{km} \mathrm{s}^{-1}$ between 10:33 and 10:38.

\subsection{Radio emission and coronal magnetic field configuration in the western hemisphere}

The bulk of the decimetric and metric radio emission after 10:20 UT came from sources in the western hemisphere which were up to $1 R_{\odot}$ away from AR 9077 . No distinct flare was seen at that time in the north-western quadrant, but the radio event was associated with perturbations in the western hemisphere which had their origin in the flaring AR 9077: EIT observed the traversal of the underlying corona by the filament ejected from the flaring active region and a large-scale perturbation which became visible near the northern pole. Figures $7 a$,b show an overlay of the radio sources (contours of equal brightness temperature) on a magnetogram and an $\mathrm{H} \alpha$ spectroheliogram taken some hours after the filament eruption and flare in AR 9077. The centroid positions of the filament inferred from EIT difference images (Figs. 3d,e) are plotted by plus-symbols connected by straight line segments. The frequency dispersion of the western sources of the metric continuum (Fig. 7a) shows that the radiating electrons were confined within a magnetic structure anchored in the leading part of AR 9077. This structure extended far to the west. If it was a closed magnetic flux tube, as suggested by the well-defined low-frequency cutoff of the continuum emission, it connected AR 9077 with regions of weak magnetic field well in the western hemisphere.

The complex of moving and stationary metre wave sources (Fig. 7b) occurred still farther away from AR 9077. In the underlying atmosphere the $\mathrm{H} \alpha$ image shows several filaments which apparently were not affected during the event, since they had similar shapes in the pre-event spectroheliograms (Kiepenheuer Institute, VTT Tenerife; Kanzelhöhe Observatory). The largest quiescent filament just north of the projected trajectory of the ejected active region filament is near $\mathrm{W} 40^{\circ}$. The photospheric magnetic field reversals inferred from the quiescent filaments and from the weak opposite polarities in the magnetogram imply the existence of large-scale coronal loops in the region between AR 9077 and the north-western limb. Faint arcades are indeed seen in the EIT images (Fig. 3). The projected trajectory of the erupting filament intersected these structures between 10:24 and 10:36 UT (cf. the middle and western position markers in Figs. 7a,b). The dimmings seen by EIT in the region where the filament had been near 10:36 (Fig. 3f) document that the magnetic field configuration was modified at this time.

In Fig. 7c the same $164 \mathrm{MHz}$ map as in b) is overlaid on images taken at 10:36 by EIT and at 10:54 by the LASCO C2 coronograph aboard SoHO (Brueckner et al. 1995). All bright white-light structures are enhanced or distorted with respect to the previous image at 10:30 UT. A conspicuous new feature is the transequatorial loop culminating about $3.6 R_{\odot}$ above the western solar limb. The loop top was just outside the $\mathrm{C} 2$ field of view in the following image (11:06 UT), and was not visible in the previous one (10:30). This is consistent with a projected speed of $\sim 1700 \mathrm{~km} \mathrm{~s}^{-1}$. We expect that the loop top was close to, but above the radio source at the time of the map in Fig. 7c. The coronal shock inferred to exist at the same time from the hectometric type II burst may 

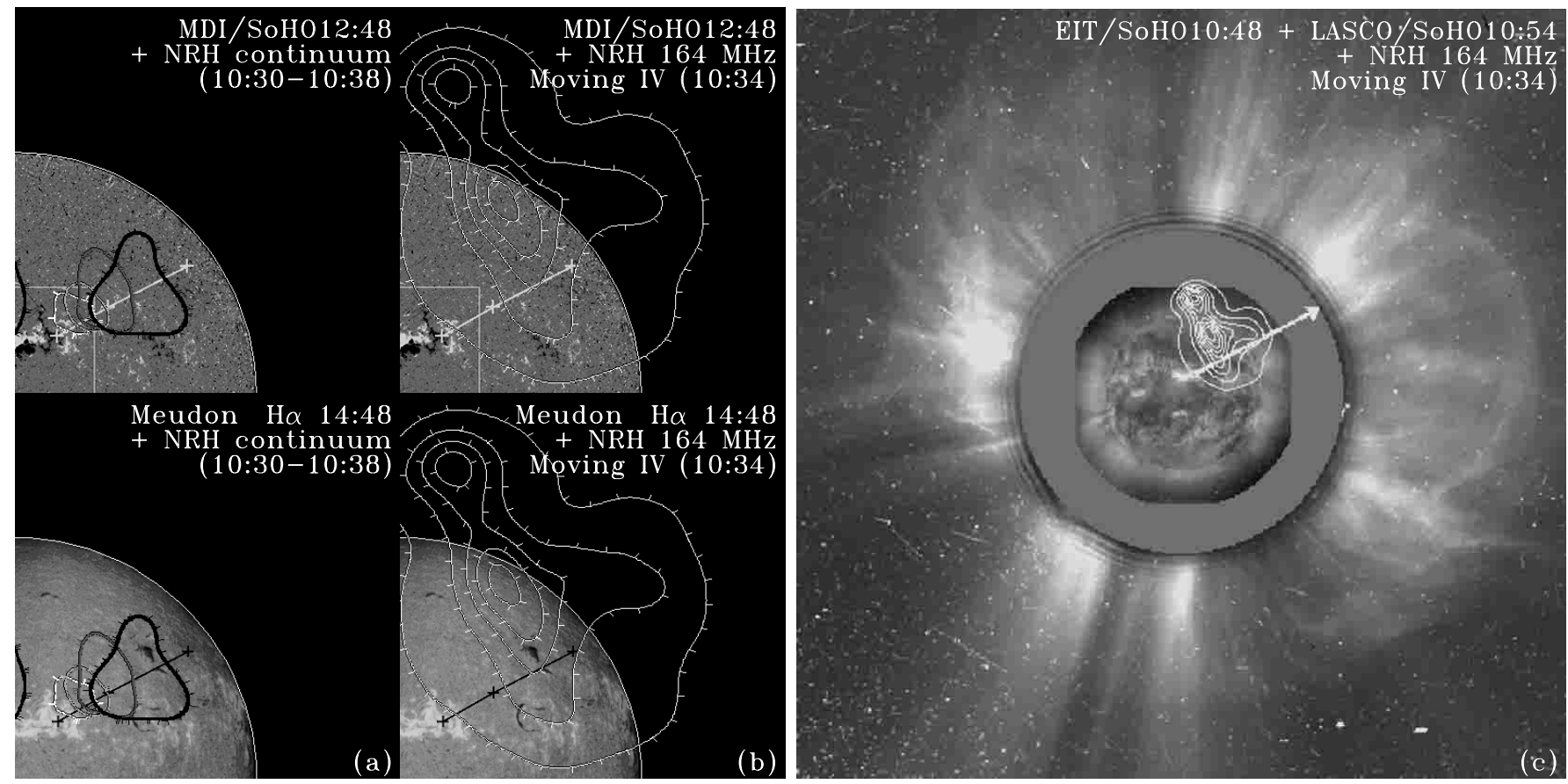

Fig. 7. Overlay of contour plots of decimetric and metric radio sources onto maps of photospheric magnetic field and coronal structures. a) Contour maps of the drifting continuum (Contours are the half-maximum levels, frequencies are 410, 327, 237, $164 \mathrm{MHz}$ in the order of increasing darkness) on top of the photospheric magnetic field map (MDI/SoHO) and an H $\alpha$ spectroheliogram (Meudon Observatory) taken several hours after the flare. The three plusses connected by straight line segments display the filament positions observed by EIT at 10:12:52, 10:24:10 and 10:36:10. b) The complex of moving and stationary type IV sources at $164 \mathrm{MHz}$ (10:34:17 UT; contours ranging from 10\% to $90 \%$ of maximum brightness) on the same MDI and H $\alpha$ maps. c) The same radio map as b) overlaid on a combined map of EIT $195 \AA$ (10:36 UT) and LASCO C2 (10:54 UT; source: LASCO homepage). The arrow extrapolates the trajectory of the ejected filament (cf. a, b)).

be the bow shock of this loop. Ambient electron densities are in the range $\left(2 \times 10^{4}-3 \times 10^{6}\right) \mathrm{cm}^{-3}$ at a height of about $3.6 R_{\odot}$ above the solar limb (cf. Koutchmy 1994, Fig. 4). Harmonic plasma emission is hence expected in the (1-16) $\mathrm{MHz}$ band, where the hectometric type II burst effectively occurred.

The arrow in the EIT image of Fig. 7c shows the extrapolated trajectory of the ejected filament. It points to the distorted structure at the northern end of the transequatorial loop. The metre wave emission was probably associated with lower coronal parts of this structure. Hence electron acceleration occurred in a localised volume within a CME. The corresponding energy release processes appear to be connected with the environment of the ejected filament and its interaction with coronal structures. The detachment of the moving type IV sources shows that the electron acceleration is related with the magnetic field reconfiguration within the CME, but on smaller spatial scale than the white-light signatures of the mass ejection.

\subsection{Summary of observational results}

The various types of electromagnetic emissions from suprathermal electrons provide evidence that during the 14 July 2000 flare and CME, electrons were accelerated over durations of several hours at different sites in the corona. The signatures of particle acceleration had different timing in different spectral ranges:

- The bulk of the hard X-rays occurred with relatively weak, although significant, decimetric to hectometric radio emission. The main radio emission was observed more than ten minutes later, while the hard X-rays exhibited a gradual fading with superposed bursts.

- The brightest hectometric type III emission, which traces the propagation of electron beams through the high corona towards interplanetary space, as well as the solar release of relativistic protons detected by neutron monitors on ground, occurred during the brightest decimetric and metric radio emission, i.e. after the prominent signatures of energy release in the low corona. They were accompanied by hectometric type II emission from a coronal shock near the starting frequency of the type III bursts.

- Radio emission from roughly $1 \mathrm{GHz}$ to $150 \mathrm{MHz}$ rose again after 10:40 UT. Metre wave emission persisted during several hours in a source connected with the trailing part of the flaring active region.

A wide range of coronal structures was involved in this activity:

- At EUV wavelengths a filament was observed to erupt in the flaring active region near central meridian and to 
propagate non-radially across the north-western quadrant. An extended loop system brightened above the location of the filament channel. The magnetic field configuration of the filament interacted with coronal structures in the north-western quadrant, far away from the flaring active region.

- At long decimetric and metric waves discrete sources brightened both in the eastern and the western hemisphere. The dominant decimetric-to-metric continuum involved two different structures in succession, which were respectively connected with the leading and the trailing part of the flaring active region. Magnetic restructuring in the north-western solar quadrant was simultaneously outlined by two moving type IV sources above the regions traversed by the filament.

- The EUV and radio phenomena were closely related in space and time to distinct features of the white-light signature of a halo CME.

\section{Discussion}

Coronal particle acceleration during the 14 July 2000 flare and CME exhibited a clear organisation in space and time. In the first $15-20 \mathrm{~min}$ of the event acceleration occurred mainly in the flaring active region near the central meridian, where hard X-ray ribbons brightened at the feet of the EUV loop arcade. Acceleration then proceeded to widespread sites around the active region, notably to the western hemisphere, where radio emission at decimetric and metric wavelengths was produced with little, if any, hard X-ray counterpart. At the same time a coronal shock wave became visible through its hectometric radio signature at greater altitude. It may be the bow shock generated by a loop which LASCO observed to rise at a speed $\geq 1700 \mathrm{~km} \mathrm{~s}^{-1}$ above the western limb.

The time history of the neutron monitor signal as a tracer of the relativistic protons at $1 \mathrm{AU}$, and the timing of the hectometric type III bursts as tracers of suprathermal electron beams in the high corona, suggest that this organisation governed also the release of particles into interplanetary space. Two weak hectometric type III bursts were the only signature that particles could escape to interplanetary space during the phase of bright hard Xray emission. The acceleration processes in the flaring active region clearly supplied particles to the low solar atmosphere, but not the relativistic protons detected at Earth. The lack of energetic charged particle injection into space during the main hard X-ray or gamma-ray emission was reported for other particle events at subrelativistic (Kallenrode \& Wibberenz 1991; Laitinen et al. 2000) and relativistic (Debrunner et al. 1997; Klein et al. 1999) energies.

The time profile of the neutron monitor signal reveals a distinct late acceleration process with impulsive character. The fast rise from background to maximum (within 10-15 min) shows that particles rapidly have access to the Earth-connected interplanetary magnetic field line. The observed profile is typical of events associated with
$\mathrm{H} \alpha$ flares between $30^{\circ}$ and $90^{\circ}$ western longitude (cf., e.g., Cliver et al. 1982), but not of a flare near the central meridian like the present one. The impulsive time profile is, however, consistent with particle injection from the acceleration sites identified in the western solar hemisphere in the radio maps on 14 July 2000. The acceleration of electrons which radiate the decimetre-metre-wave continuum in the western hemisphere started near the inferred onset of the relativistic proton injection and was turned off after about $10 \mathrm{~min}$. Thereafter electron acceleration continued in the middle corona, but in the eastern hemisphere in relationship with the trailing part of the active region. The electron acceleration in the western hemisphere was associated with the restructuring of the magnetic field there, as documented by the moving radio sources and the changes in the underlying corona related to the passage of the filament. Hence a scenario where suprathermal electrons and relativistic protons which escape to $1 \mathrm{AU}$ are accelerated in association with the restructuring of the magnetic field in the western hemisphere is consistent with the white-light, EUV and radio imaging observations, and readily accounts for the late onset and rapid rise of the neutron monitor signal. From the observations (i) that quiescent filaments in the vicinity of the radio sources were not affected by these perturbations and (ii) that the radio continuum extended down to about $150 \mathrm{MHz}$, we infer that the magnetic restructuring and particle acceleration occurred in the height range $(0.1-1) R_{\odot}$ above the photosphere.

An alternative to acceleration in the magnetically stressed corona is the bow shock of the CME. The bow shock is a plausible exciter of the hectometric type II burst. The start of this shock signature is simultaneous with the processes of reconfiguration in the middle corona and of electron acceleration revealed by the decimetricmetric radio emission, and with the release of relativistic protons. However, acceleration at the bow shock offers no explanation for the impulsive time profile of the neutron monitor signal. Cane et al. (1988) (see also Reames 1999) grouped the time profiles of solar particle events in three categories, explaining fast rises by shock acceleration on the well-connected field line at rather low coronal heights, and slow rises by delayed acceleration when a shock driven by a CME near central meridian or in the eastern solar hemisphere eventually intersects the Earth-connected interplanetary field line. A fast halo CME such as 14 July 2000 is expected to generate both the rapidly and the slowly rising component, i.e. a rather flat time profile or a long rise. This is clearly not consistent with the observations. A similar discrepancy arises when the time profile of 14 July 2000 is compared with other GLEs. Kahler (1994) discussed three GLEs where the neutron monitor signal from protons $\geq 1 \mathrm{GeV}$ rose while the CME front travelled from $4 R_{\odot}$ to $\geq 12 R_{\odot}$ above the limb (cf. Fig. 5 of Kahler 1994). Kahler supposed that the relativistic protons were accelerated by the bow shock of the CME, and concluded on the basis of this (unproven) assumption that this happened continuously while the CME travelled through this 
height range. On 14 July 2000 the maximum of the neutron monitor signal at Mount Wellington was attained before the time of the LASCO image in Fig. 7 , at only $4 R_{\odot}$ above the photosphere. The associated CME was as fast $\left(1700 \mathrm{~km} \mathrm{~s}^{-1}\right)$ as those of Kahler's sample. From the arrival of the shock at SoHO about 28 hours later (e.g., CELIAS measurements of the solar wind speed, SoHO homepage) the mean transit speed of the Earth-directed part of the shock $\left(1500 \mathrm{~km} \mathrm{~s}^{-1}\right)$ was not much smaller than the plane-of-the-sky speed of the CME in the corona. There is hence no obvious reason why the relativistic proton acceleration should cease so much earlier on 14 July than in Kahler's sample if the CME shock was the main accelerator.

Coronal magnetic restructuring during the 14 July 2000 event provides a ready explanation for both the onset and the rise time of the neutron monitor signal. The relevant processes could be observed thanks to the favorable configuration of the flaring active region and the trajectory of the ejected magnetic structure, and because highly complementary particle and remote sensing measurements were available. We suggest that similar effects act in other GLEs. Indeed, one of Kahler's GLEs is the 29 September 1989 event which Klein et al. (1999) showed to have a similar late relativistic proton injection associated with a decimetric-metric continuum as 14 July 2000. Such common features between decimetric-to-metric radio emission and relativistic proton time profiles suggest that the bow shock of a CME may not be the predominant accelerator of relativistic protons in the corona. This does not exclude that the shock is actually present in the corona during the acceleration. But we note that, although the presence of a type II burst was often considered as evidence for shock acceleration of simultaneously observed electrons (e.g., Cane et al. 1981; Mann et al. 1999), previous investigations suggest that such an association may be misleading for highenergy and relativistic particles (Hucke et al. 1992; Klein \& Trottet 1994, 2001; Reiner et al. 2000; Laitinen et al. 2000).

The identification of coronal acceleration sites of escaping particles thus does not imply that the CME plays no role in the particle event. In the 14 July 2000 event there is evidence that magnetic field reconfiguration far from the flaring active region is crucial for the production and injection of particles detected at $1 \mathrm{AU}$. A plausible driver of these changes is the ejected magnetic field configuration around the filament, which is part of the CME.

Acknowledgements. The authors are very much indebted to colleagues who generously supplied data for this work: C. Alissandrakis (Athens, Artemis spectrograph), F. Farnik (Prague, HXRS satellite), M. Kaiser (Greenbelt, WAVES/WIND), J. Khan \& T. Kosugi (Tokyo, YohkohHXT), P. Zlobec (Trieste, decimetric radio data). TRACE, LASCO/SoHO and MDI/SoHO images and movies and radio spectra of the Ondřejov (Prague) and IZMIRAN (Moscow) spectrographs were obtained via the instrument homepages and the ISTP homepage. GOES data were provided by the Solar Data Analysis Center at NASA/GSFC. The neutron monitors at Terre Adélie and Kerguelen are operated by the French Polar Institute (IFRTP), those at Hobart and Mount Wellington by the Department of Physics, University of Tasmania. Data were provided by the database of the GLE Project, Geophysics Directorate/PL, Hanscom AFB, USA. KLK acknowledges helpful discussions with G. Aulanier, W. Droege and L. Fletcher. SoHO is a project of international cooperation between ESA and NASA. The Yohkoh mission is a project of the Institute of Space and Astronautical Science (ISAS) of Japan. The Nançay Radio Observatory is funded by the French Ministry of Education, the CNRS and the Région Centre.

\section{References}

Akimov, V. V., Ambrož, P., Belov, A. V., et al. 1996, Solar Phys., 166, 107

Brueckner, G. E., Howard, R. A., Koomen, M. J., et al. 1995, Solar Phys., 162, 357

Cane, H. V., Reames, D. V., \& von Rosenvinge, T. T. 1988, JGR, 93, 9555

Cane, H. V., Stone, R. G., Fainberg, J., et al. 1981, Geophys. Res. Lett., 8, 1285

Carmichael, H. 1962, Space Sci. Rev., 1, 28

Caroubalos, C., Maroulis, D., Patavalis, N., et al. 2001, Exper. Astron., in press

Cliver, E. W., Kahler, S. W., Shea, M. A., \& Smart, D. F. 1982, ApJ, 260, 362

Debrunner, H., Lockwood, J. A., Barat, C., et al. 1997, ApJ, 479, 997

Delaboudinière, J. P., Artzner, G. E., Brunaud, J., et al. 1995, Solar Phys., 162, 291

Fox, N. 2000, in EOS, Transactions AGU, 81(48), F968

Hucke, S., Kallenrode, M. B., \& Wibberenz, G. 1992, Solar Phys., 142, 143

Kahler, S. 1994, ApJ, 428, 837

Kallenrode, M., \& Wibberenz, G. 1991, ApJ, 376, 787

Karlický, M., Yan, Y., Fu, Q., et al. 2001, A\&A, 369, 1104

Kerdraon, A., \& Delouis, J. 1997, in Coronal Physics from Radio and Space Observations, ed. G. Trottet, LNP No. 483 (Springer), 192

Klein, K. L., Chupp, E. L., Trottet, G., et al. 1999, A\&A, 348, 271

Klein, K.-L., \& Trottet, G. 1994, in High-Energy Solar Phenomena - a New Era of Spacecraft Measurements, ed. J. Ryan, \& W. Vestrand, AIP Conf. Proc., 294, 187

Klein, K.-L., \& Trottet, G. 2001, Space Sci. Rev., 95, 215

Kocharov, L. G., Kovaltsov, G. A., Kocharov, G. E., et al. 1994, Solar Phys., 150, 267

Kosovichev, A. G., \& Zharkova, V. V. 2001, ApJ, 550, L105

Koutchmy, S. 1994, Adv. Space Res., 14(4), 29

Laitinen, T., Klein, K. L., Kocharov, L., et al. 2000, A\&A, 360, 729

Lockwood, J. A., Debrunner, H., \& Flueckiger, E. O. 1990, JGR, 95, 4187

Mann, G., Classen, H. T., Motschmann, U., Kunow, H., \& Dröge, W. 1999, Astrophys. Spa. Sci., 264, 489

Miroshnichenko, L. I., De Koning, C. A., \& Perez-Enriquez, R. 2000, Space Sci. Rev., 91, 615

Palmer, I. D., \& Smerd, S. F. 1972, Solar Phys., 26, 460

Reames, D. V. 1999, Space Sci. Rev., 90, 413

Reiner, M. J., Karlický, M., Jiřička, K., et al. 2000, ApJ, 530, 1049

Title, A. 2000, in EOS, Transactions AGU, 81(48), F968

Yan, Y., Deng, Y., Karlický, M., et al. 2001, ApJ, 551, L115 